\title{
Whole-exome sequencing identifies a somatic missense mutation of $N B N$ in clear cell sarcoma of the salivary gland
}

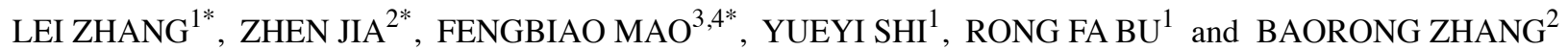 \\ ${ }^{1}$ Department of Stomatology, Chinese PLA General Hospital, Beijing 100853; ${ }^{2}$ Department of Stomatology, \\ Beijing Aviation General Hospital, Beijing 100012; ${ }^{3}$ Beijing Institutes of Life Science, Chinese Academy of Sciences, \\ Beijing 100101; ${ }^{4}$ University of the Chinese Academy of Sciences, Beijing 100049, P.R. China
}

Received December 12, 2015; Accepted January 15, 2016

DOI: $10.3892 / o r .2016 .4738$

\begin{abstract}
Clear cell sarcoma (CCS) is a rare, low-grade carcinoma commonly located in the distal extremities of young adults involving tendons and aponeuroses. CCS is characterized by its poor prognosis due to late diagnosis, multiple local recurrence, propensity to late metastases, and a high rate of tumor-related mortality. The genetic cause for CCS is thought to be EWSRI gene translocation. However, CCS lacking a translocation may have other, as yet uncharacterized, genetic mutations that can cause the same pathological effect. A combination of whole-exome sequencing and Sanger sequencing of cancer tissue and venous blood from a patient diagnosed with CCS of the salivary gland revealed a somatic missense mutation, c.1061C > T (p.P354L), in exon 9 of the Nibrin gene $(N B N)$. This somatic missense mutation led to the conversion of proline to leucine (p.P354L), resulting in deleterious effects for the NBN protein. Multiple-sequence alignments showed that codon 354 , where the mutation (c.1061C $>\mathrm{T}$ ) occurs, is located within a phylogenetically conserved region. In conclusion, we here report a somatic missense mutation c.1061C $>\mathrm{T}$ (p.P354L) in the $N B N$ gene in a patient with CCS lacking an EWSR1-ATF1 fusion. Our findings broaden the genotypic spectrum of CCS and provide new molecular insight that should prove useful in the future clinical genetic diagnosis of CCS.
\end{abstract}

\section{Introduction}

Clear cell sarcoma (CCS), regarded as malignant melanoma of soft parts, is a rare aggressive tumor that accounts for less than

Correspondence to: Dr Rong Fa Bu, Department of Stomatology, Chinese PLA General Hospital, Beijing 100853, P.R. China

E-mail: rongfabu@aliyun.com

Dr Baorong Zhang, Department of Stomatology, Beijing Aviation General Hospital, Beijing 100012, P.R. China

E-mail: zhangbaorongbj@163.com

${ }^{*}$ Contributed equally

Key words: NBN gene, clear cell sarcoma, salivary gland, exome sequencing
$1 \%$ of all soft tissue sarcomas $(1,2)$. CCS is characterized by its poor prognosis due to late diagnosis, multiple local recurrence, propensity to late metastases, and a high rate of tumor-related mortality $(3,4)$. As one of the few sarcomas with a high propensity for lymph node metastases (5), CCS is a locally aggressive neoplasm with a high rate of recurrence and metastasis (more than $50 \%)(2,6,7)$. The 5 -year disease-specific survival rates have been reported to be approximately 50-67\%, but these values are not representative of long-term survival since many patients develop lung and bone metastases more than five years after initial resection $(7,8)$. This tumor is observed most frequently in young adults and predominantly affects the soft tissues of the distal extremities, with the majority being deep seated and involving tendons and aponeuroses $(4,9)$. Occasionally, it can arise in visceral organs including the gastrointestinal tract (10). It is rarely seen in the head or neck, with for example only $1.2 \%$ of the approximately 500 reported cases of CCS currently involving the head or neck (1).

Clear cell sarcoma was first described by Dr Franz M. Enzinger in 1965 (11). CCS is characterized by a nested or fascicular growth pattern of spindled and epithelioid cells with clear or lightly eosinophilic cytoplasm surrounded by fibrous septa (12). The tumor cells have elongated oval nuclei with prominent nucleoli and occasional nuclear pseudo-inclusions (13). Multinucleated giant cells are identified in more than half of the reported cases (7). Uniquely among primary soft tissue tumors, pre-melanosomes are present in almost all cases of CCS, detectable by electron microscopy (14). As a result, immunohistochemistry (IHC)-based tests of CCS cells are almost always positive for the melanoma markers S-100, HMB45, MelanA, and microphthalmia transcription factor (MITF), although melanin staining is not always observed $(12,15)$. Adverse prognostic factors for CCS identified to date include large tumor size and any microscopic tumor necrosis. Surgery is the mainstay of treatment for this high grade sarcoma, with chemotherapy having little effect. Although the melanocytic differentiation of CCS is indisputable, its precise lineage remains unclear. Thus, CCS maintains the status of a unique yet enigmatic clinicopathological entity (4).

As a rare type of soft tissue sarcoma, CCS exhibits morphological, immunohistochemical and ultrastructural similarity with malignant melanoma (5). CCSs share many 
features with malignant melanoma, including expression of melanoma markers (16). However, in contrast to most melanomas, CCSs lack BRAF mutations (9). In addition, molecular analysis has revealed that CCSs are distinct tumors; they present the specific $\mathrm{t}(12 ; 22)(\mathrm{q} 13 ; \mathrm{q} 12)$ translocation that results in the chimeric gene EWSR1/ATF1, which is not observed in melanomas (5). The genetic cause for CCS is considered to be this defining gene translocation. Previous cytogenetic studies have established the specificity of the recurrent $t(12 ; 22)$ (q13;q12) translocation, resulting in an EWSR1-ATF1 fusion for CCS (17). However, EWSR1-ATF1 fusion derived from cytogenetic rearrangements is characteristic but not entirely unique for CCS, as similar fusion genes are also present in angiomatoid fibrous histiocytoma (18). Generally, detection of this fusion gene and the absence of BRAF gene mutations easily distinguish CCS from cutaneous melanoma (4).

Having said that, it must be noted that not all CCSs present with EWSR1 rearrangements. According to a previous study, approximately $70 \%$ of CCS cases harbored a rearrangement (EWSR1-ATF1 or EWSR1-CREB1) in the EWSR1 locus with a mean of $81.6 \%$ positive cells/sample (range, 60-95\%) (19). The more prevalent fusion event in CCS, EWSR1-ATF1, also occurs in both hyalinizing clear cell carcinoma (20) and angiomatoid fibrous histiocytoma (18). However, the exons involved are different, with most CCS tumors involving EWSR1 exons 7, 8, or 10 fused to ATF1 exons 4, 5, or 7 (21), but with HCC tumors harboring EWSR1 exon 11 fused in-frame to exon 3 of ATF1 (20). Therefore, the specific EWSR1-ATF1 fusion in CCS can typically be used to distinguish CCS from its mimics such as spindle cell melanoma, spindle cell squamous carcinoma, cutaneous leiomyosarcoma and atypical fibroxanthoma, as well as from other tumors with melanocytic differentiation (12).

To date, the pathogenesis of CCS lacking an EWSR1 rearrangement remains poorly characterized. No somatic mutations involved in CCS have been identified, but single nucleotide variants are known to play a significant role in tumorigenesis (22). In the present study, we identified a somatic missense mutation c.1061C >T (p.P354L) in exon 9 of the Nibrin $(N B N)$ gene in a patient with CCS of the salivary gland via a combination of exome sequencing and Sanger sequencing. It is known that although mutations of $N B N$ do not play a major role in predisposition to melanoma of the skin, alterations in this gene may contribute to the risk for breast cancer (23-25).

NBN is a protein associated with the repair of double-strand breaks (DSBs), which cause serious damage to genomes. NBN is a 754 amino acid protein known to be a member of the NBS1/hMre11/RAD50 double-strand DNA break repair complex (referred to as MRN) (26). This complex recognizes DNA damage and rapidly relocates to DSB sites, forming nuclear foci. NBN also has a role in the regulation of MRN protein complex activity, including involvement in end-processing of both physiological and mutagenic DNA DSBs. The mutations within exons 6-10 of the $N B N$ gene in patients suffering Nijmegen breakage syndrome (NBS) result in a truncated protein (27). Patients with NBS are predisposed to cancers. This predisposition to cancer may be linked to the DSBs that occur during the development of lymphoid cells. Moreover, mutations of NBN have been reported to be associated with many types of cancers, including gastrointestinal lymphoma (28), childhood acute leukaemia (29), glioblastomas (30), and breast cancer (25). In this study, we report the first known somatic mutation of NBN that was found to be involved in CCS of the salivary gland, and provide molecular insight into future clinical genetic diagnosis for CCS by broadening the genotypic spectrum of CCS.

\section{Materials and methods}

Subject and statement of ethics. The subject was a 20 -year-old Chinese male that presented with a gradual mass of duration 3-4 months located on the left parotid gland. The mass was diagnosed as clear cell sarcoma (CCS) of the salivary gland by incisional biopsy.

The experimental methods used in this study were carried out in accordance with the relevant guidelines and regulations. This study was approved by the Ethics Committee of the General Hospital of the Chinese People's Liberation Army. Relevant informed consent was obtained from all participants, including the CCS patient and the healthy control subjects.

All sequencing data for the tumor and venous blood samples have been deposited in the Sequence Read Archive (SRA, http://www.ncbi.nlm.nih.gov/sra/) with accession nos. BioSample SAMN03384326 and SAMN03384334, respectively, in BioProject SRP055838.

Exome sequencing. The tumor and venous blood from the Chinese male CCS patient was selected for exome sequencing. Exome sequencing was carried out using an Agilent SureSelect Human All Exon v5.0 (51M) kit, according to the instructions from Illumina's TruSeq Exome Enrichment Guide (SureSelectXT Target Enrichment System for Illumina Paired-End Sequencing Library, Agilent). Genomic DNA libraries were prepared according to the manufacturer's instructions (Illumina Inc., USA). Briefly, $3 \mu \mathrm{g}$ of genomic DNA was randomly fragmented into pieces of 100-500 bp in size using a Diagenode Bioruptor ${ }^{\circledR}$ system (Diagenode). DNA fragments between 150 and 250 bp were recovered by gel extraction. An end repair and size selection procedure was then performed with T4 DNA polymerase and Klenow polymerase cleavage 3 '. An 'A' base was added to the 3 ' end of the fragments using Klenow 3 ' to 5 ' exo minus. The DNA fragments were then ligated to the Illumina multi-PE-adaptor. The adapter-ligated templates were purified using Agencourt AMPure SPRI beads and amplified by four-cycle ligationmediated polymerase chain reaction (LM-PCR), under the following PCR conditions: $2 \mathrm{~min}$ at $94^{\circ} \mathrm{C}$, four cycles of $10 \mathrm{sec}$ at $94^{\circ} \mathrm{C}, 30 \mathrm{sec}$ at $62^{\circ} \mathrm{C}$, and $30 \mathrm{sec}$ at $72^{\circ} \mathrm{C}$, and then $5 \mathrm{~min}$ at $72^{\circ} \mathrm{C}$. The LM-PCR products were hybridized to the Agilent Oligo pool for $24 \mathrm{~h}$ at $65^{\circ} \mathrm{C}$ for enrichment. The hybridized fragments were bound to streptavidin beads and non-hybridized fragments were washed out. Captured LM-PCR products were amplified by PCR $\left(2 \mathrm{~min}\right.$ at $98^{\circ} \mathrm{C} ; 10-12$ cycles for $10 \mathrm{sec}$ at $98^{\circ} \mathrm{C}, 30 \mathrm{sec}$ at $60^{\circ} \mathrm{C}, 30 \mathrm{sec}$ at $72^{\circ} \mathrm{C}$, followed by $5 \mathrm{~min}$ at $72^{\circ} \mathrm{C}$ ). The magnitude of enrichment of the samples was estimated with an Agilent 2100 bioanalyzer. The captured library was then sequenced on Illumina HiSeq 2000 analyzers with 126 cycles/read, to generate paired-end reads and $8 \mathrm{bp}$ of index tag (following the manufacturer's standard sequencing instructions). 

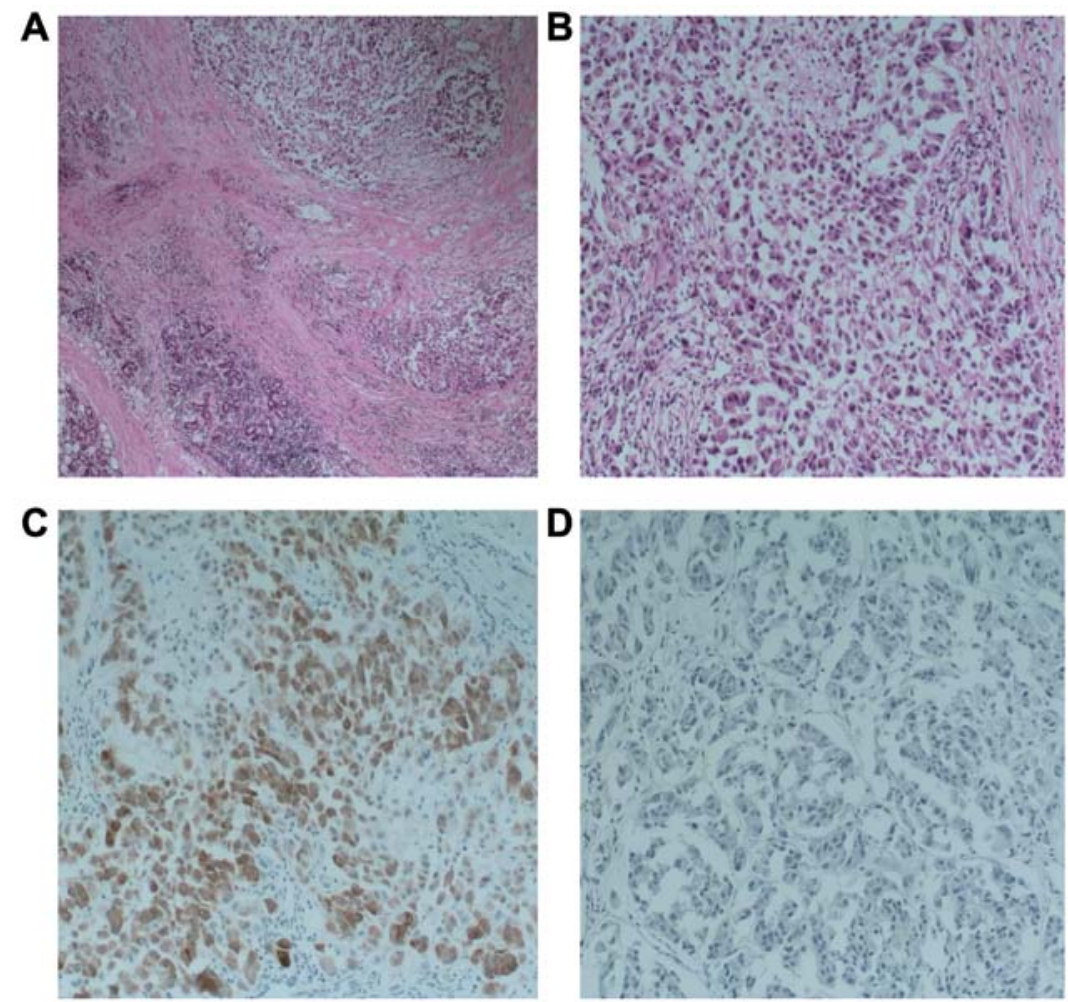

Figure 1. Diagnosis of clear cell sarcoma (CCS) of the salivary gland by incisional biopsy. (A) Sub-mucosal mass with normal epidermis and an ill-defined infiltrative process present in the reticular dermis are observed (x100 magnification, H\&E). (B) Cytoplasmic infiltrate composed of spindled eosinophilic cells with haphazard arrangement and intersecting dense collagen bands is present in clearing for CCS of the salivary gland (x200 magnification, H\&E) (C) Immunohistochemical staining for HMB45 is positive in tumor cells (x100 magnification, immunohistochemistry). (D) Immunohistochemical staining for $\mathrm{CK}$ is negative in tumor cells (x200 magnification, immunohistochemistry).

Read mapping and variant analysis. Image analysis and base calling were performed with Illumina Basecaller program (v1.8). Indexed primers were used for data fidelity surveillance. The sequence reads were aligned to the human genome reference obtained from the UCSC database (http://genome. ucsc.edu/), version hg19 (GRCh37), using the SOAP aligner program (v2.21). Single nuclotide polymorphisms (SNPs) were called using SOAPsnp (v1.03) with the default parameters, after the duplicated reads (produced mainly in the PCR step) had been removed using Picard (v1.63) (31). Short insertions or deletions (InDels) altering coding sequence or splicing sites were identified by GATK (v1.4-33-g051b450) through realignment analysis of insertions and deletions, quality recalibration, and InDels calling (UnifiedGenotyper in GATK). We then filtered candidate SNPs with the following criteria: SNP quality, $\geq 20$; sequencing depth, $\geq 10$; the estimated copy number, $\leq 2$; and the distance between two SNPs, $>5$. Subsequently, we used VarScan2 v2.3.7 (http://varscan.sourceforge.net/) to detect the somatic mutations in the exome data from tumor-normal pairs. We applied varElect (http://varelect. genecards.org/) to select mutations associated with the function of salivary glands. The effect of candidate mutations to protein features were predicted with the GERP++ program (May 22, 2011). Potential rejected substitutions were evaluated by PolyPhen-2 (http://genetics.bwh.harvard.edu/pph2/) and SIFT prediction (http://sit.jcvi.org/).

PCR and Sanger sequencing. Following the analysis of the exome sequencing data, Sanger sequencing was performed to verify the detected genetic variants. Primers flanking the mutation area of NBN (NM_002485) were designed based on the reference genomic sequences of the human genome from NCBI GenBank, and synthesized in Shanghai, China, by Thermo Scientific. The sequence of the forward primer was 5'-CCCTACCTCATTGGCTTTGTG-3', and that of the reverse primer was 5'-TATCACGGTCCCTGCTTCC-3'. All PCR amplification was carried out using an Applied Biosystems Life Technologies (ABI) 9700 thermal cycler. PCR products were directly sequenced on an ABI PRISM 3730 automated sequencer (Applied Biosystems Life Technologies). Sequence comparisons and analyses were performed using the Jalview program (v2.8.2; http://www.jalview.org/).

\section{Results}

Clinical phenotype. A 20-year-old male presented with a gradual mass of duration of 3-4 months on the left parotid gland. He occasionally suffered from a short period of needle pain. On palpation, a sub-mucosal mass measuring $3 \times 2 \times 2 \mathrm{~cm}$ in size was confirmed. The mass was non-tender and varied in consistency from soft to firm. On general examination, the patient appeared apparently healthy with neither submandibular nor cervical lymph node metastases. The mass was diagnosed as CCS of the salivary gland by incisional biopsy (Fig. 1A-D). Histologically, the tumor had typical features including a combination of cords and nests of clear and eosinophilic cells in a hyalinized background (Fig. 1B). However, there was a relatively wide range of features in the tumor cells. Most of the 
A Tumor tissue

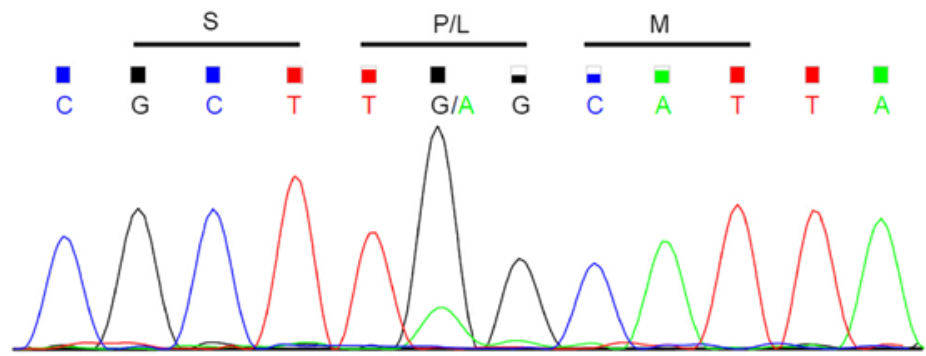

B Venous blood

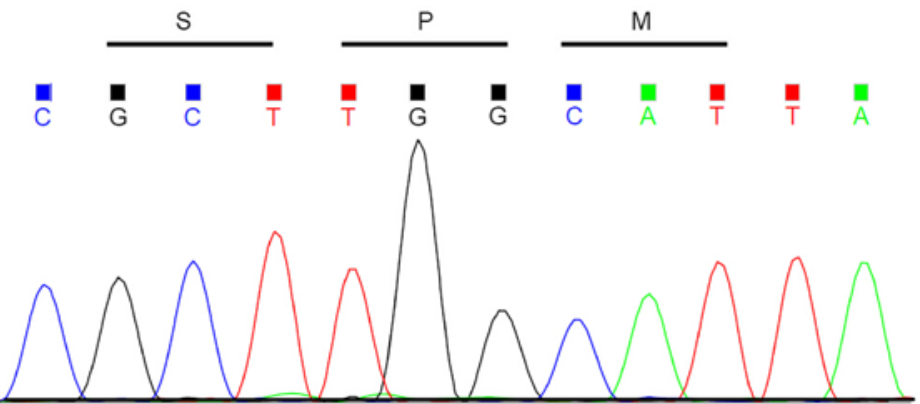

Figure 2. DNA sequence chromatograms of Sanger sequencing. (A and B) A missense mutation in exon 9, c.1061C $>$ T, results in p.P354L in the tumor tissue (A) and in its matched blood sample (B).

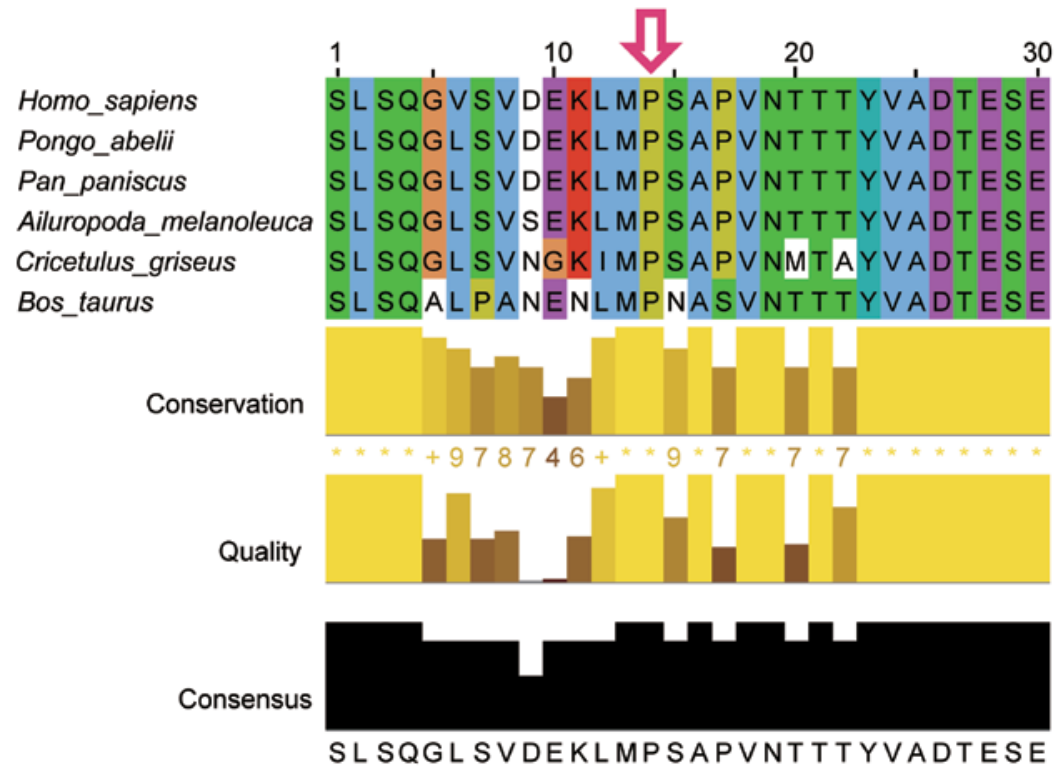

Figure 3. Conservation analysis of the p.Pro354 amino acid residue of the NBN protein.

tumor cells actually had a pale eosinophilic cytoplasm rather than a clear cytoplasm, or they may have had a mixture of both (Fig. 1A). Focal squamous differentiation was also noted occasionally (Fig. 1C). There was a tendency for the cells in the center of the mass to be admixed with, or to be surrounded by, a hyalinized basement membrane-like material (Fig. 1D). Tumor cells at the periphery of the mass had a greater tendency for nest formation and for wide infiltration without a desmoplastic response or stromal deposition (Fig. 1A). Moreover, immunohistochemical staining revealed that the tumors were HMB45-positive (Fig. 1C) and CK-negative (Fig. 1D). Based on these observations and lines of evidence, we diagnosed this tumor as CCS of the salivary gland.
Mutation analysis. We performed exome sequencing of the tumor tissue from the CCS patient. We generated 19.28 and 7.95 billion bases of 125-bp paired-end read sequences from the tumor and from the venous blood samples, respectively. Billion bases [19.09 (99\%) and 7.88 (99\%)] passed the quality assessment, and $19.01(98.61 \%)$ and 7.91 (99.46\%) billion bases aligned to the human reference for the tumor and venous blood samples, respectively. For tumor tissue, 11.39 billion bases $(59.9 \%)$ mapped to the targeted regions with a mean coverage of 80.47 X 21,077 genetic variants, including 9,295 non-synonymous variants, were identified in either the coding regions or the splice sites. For venous blood, 4.61 billion bases $(58.3 \%)$ mapped to the targeted regions 


\section{chr8}

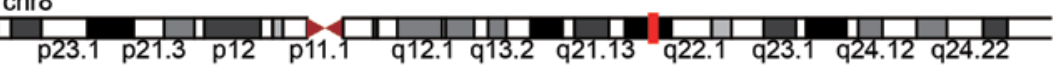

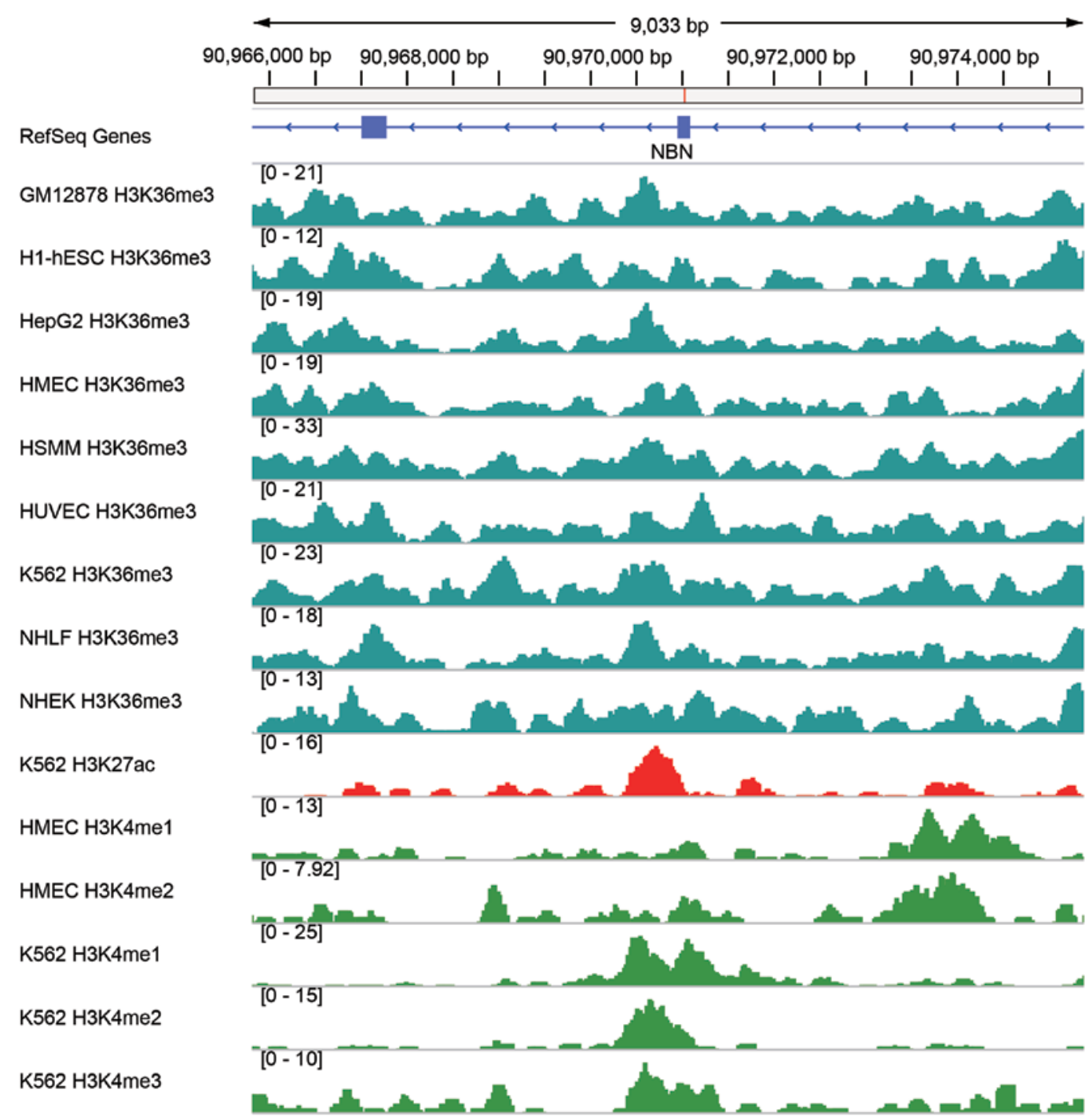

Figure 4. A snapshot of histone modification from the ENCODE project in the locus around the NBN mutation, as viewed with the Integrative Genomics Viewer tool.

with a mean coverage of $63.42 \mathrm{X} ; 19,930$ genetic variants, including 8,404 non-synonymous variants, were identified in either the coding regions or the splice sites. A prioritization scheme was applied to identify the pathogenic mutation in the tumor tissue, using methods similar to those reported in recent studies $(32,33)$. We excluded the known variants that had been identified in the dbSNP141, 1000 genomes, HapMap, and ESP-6500 datasets. After filtering the variants displayed in venous blood, we obtained 542 candidate somatic mutations in the tumor tissue.

Subsequently, we used varElect software to select mutations associated with the function of the salivary gland and acquired four candidate causal mutations. A somatic mutation, c.1061C $>\mathrm{T}$ (p.P354L), was eventually identified in exon 9 of the $N B N$ gene in the tumor and this was validated using Sanger sequencing. This mutation results in a missense variant. The same mutation was absent in peritumoral tissue of the patient (Fig. 2) and venous blood samples from 30 ethni- cally matched normal control individuals. This mutation was also absent in the dbSNP141, 1000 genomes, HapMap, and ESP-6500 datasets. To evaluate whether or not there were EWSR1-ATF1 or EWSR1-CREB1 gene translocations, RT-PCR (reverse transcription polymerase chain reaction) was performed to validate the fusion site; the negative result was thus confirmed.

Bioinformatic analysis of NBN mutations in CCS. We obtained sequences for NBN family proteins using the BLAST tools of the NCBI databases and performed multiple-sequence alignments using Jalview in various animal species, including Homo sapiens, Pongo abelii, Pan paniscus, Ailuropoda melanoleuca, Cricetulus griseus and Bos taurus (Fig. 3). The p.Pro354Leu variant was found to be located in a highly conserved region of the NBN protein, suggesting its likely structural and functional importance. This mutation was predicted to affect the protein features and be rejected 

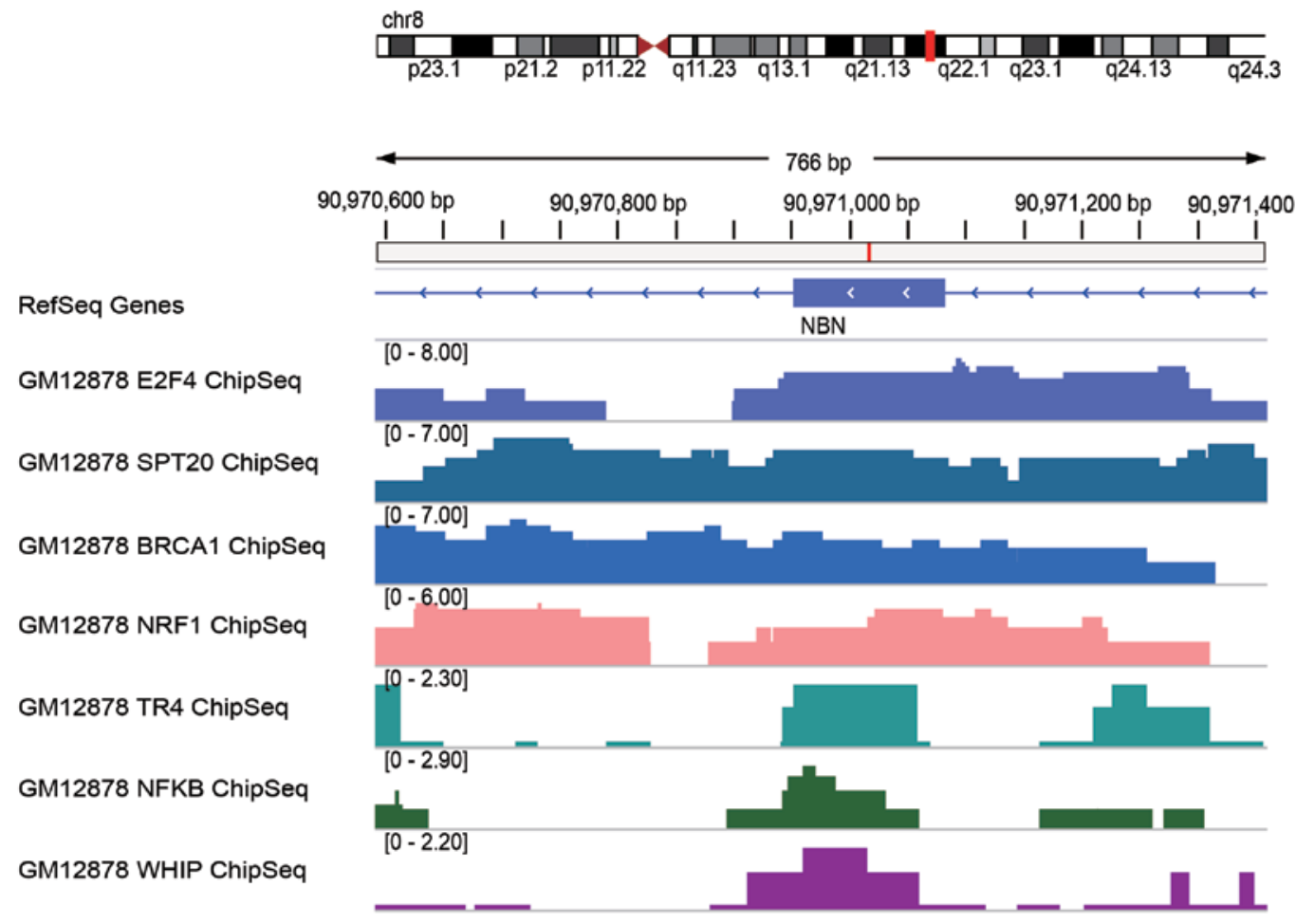

Figure 5. A snapshot of transcriptional factors of from the ENCODE project in the locus around the NBN mutation as viewed with the Integrative Genomics Viewer tool.

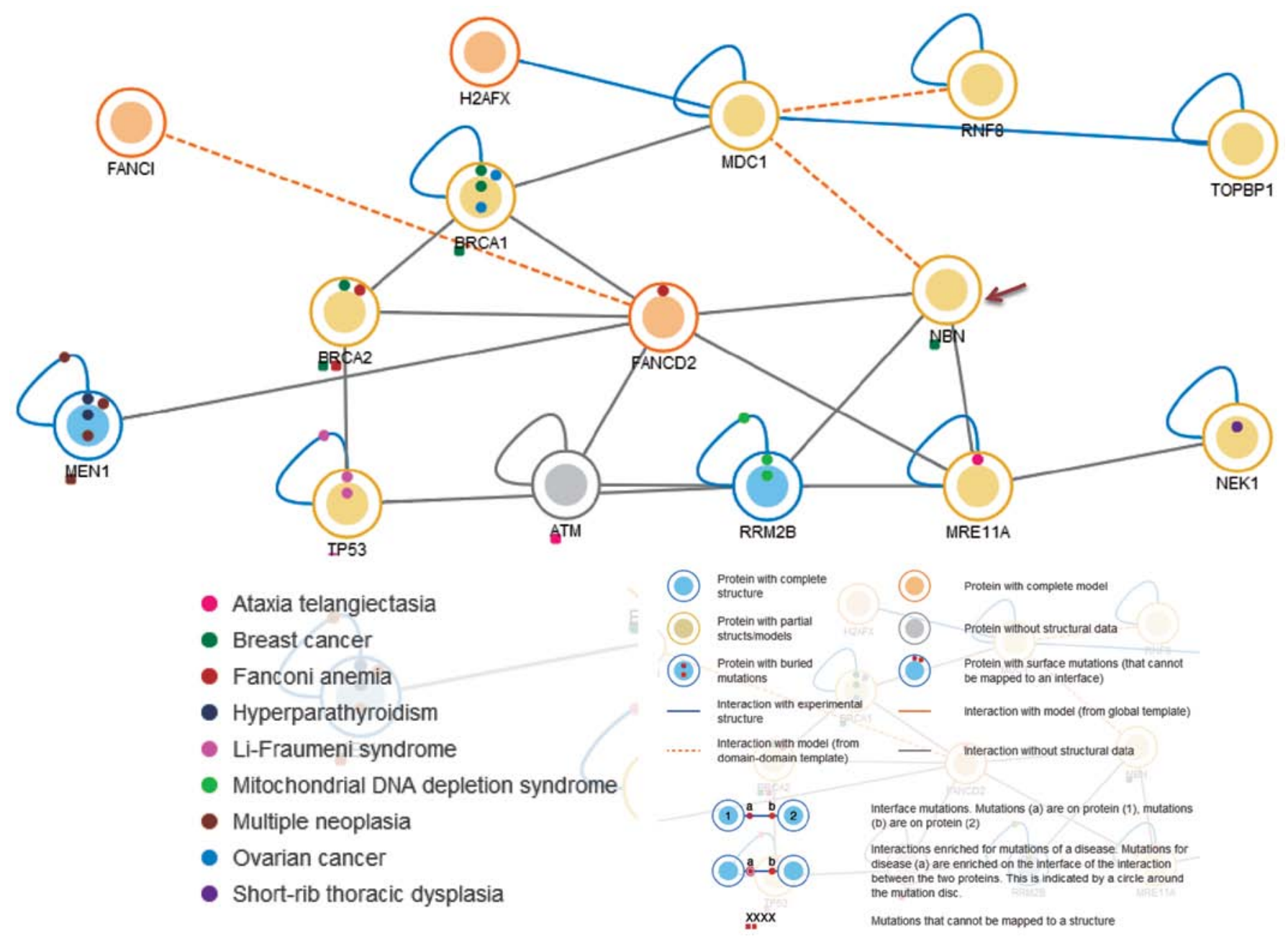

Figure 6. Network analysis of NBN using the dSysMap ('Disease-mutations Systemic Mapping') program. 
substitutions predicted by GERP++ with a score 3.07. SIFT prediction indicated a deleterious effect for this mutation, with a score of 0 . In addition, PolyPhen- 2 prediction also suggested that this mutation probably conferred a damaging effect, with a confident score of 0.998 .

GO annotations related to NBN include damaged DNA binding and transcription factor binding. However, it remains unclear which histone modifications regulate the expression of $\mathrm{NBN}$ and it is not yet clear which transcriptional factors are associated with the mutation locus of NBN. Using integrated analysis of the data for histone modifications deposited in the Encyclopedia of DNA Elements (ENCODE) project (34), we found that histone modifications at the NBN locus and the c. $1061 \mathrm{C}>\mathrm{T}$ mutant variant include H3K36me3, H3K27ac, and $\mathrm{H} 3 \mathrm{~K} 4 \mathrm{mel} / 2 / 3$ in multiple cell lines such as GM12878, H1-hESC and K562 (Fig. 4). As these histone modifications are canonically active molecule markers (35), we speculated that the $\mathrm{c} .1061 \mathrm{C}>\mathrm{T}$ variant may influence histone modification and affect the expression of NBN, although it must be noted that the histone modification data are from cell lines rather than from CCS tumors. Indeed, according to our PCR validation of expression analysis, the expression of NBN was decreased in the CCS tumor compared with its expression level in peritumoral tissue. Similarly, several transcriptional factors deposited in the ENCODE project were found to be enriched by ChIP-seq in the locus where the c.1061C $>\mathrm{T}$ variant occurred in the $N B N$ gene for cell line GM12878 (Fig. 5). These transcriptional factors were identified as E2F4, SPT20, BRCA1, NRF1, TR4, NFKB and WHIP; these may function to regulate the transcription of the $N B N$ gene.

\section{Discussion}

Clear cell sarcoma (CCS) of soft tissue, formerly referred to as malignant melanoma of soft parts, is a neoplasm with poor prognosis that primarily affects young adults between the ages of 20 and 40 years (36). The tumor has a high propensity for lymph node metastasis and local recurrence. CCS typically involves tendons and aponeuroses. Primary CCS of the salivary glands is exceedingly rare. Only a few cases of primary CCS arising in the ulna, metatarsals, ribs, radius, sacrum, humerus (37) and jejunum (10) have been reported, and to the best of our knowledge, our case of CCS arising in the salivary glands is only the second such study to date.

CCS is a translocation-associated sarcoma. In chromosomal translocations, the pieces of two chromosomes are swapped; this can result in an abnormal fusion of genes. Most cases of CCS harbor a fusion of EWSR1/ATF1 resulting from translocation (21). The genetic cause for CCS is thought to be its defining gene translocation. However, $30 \%$ of cases of CCS have no observed EWSR1 translocation (10). A CCS lacking a translocation may have other, as yet uncharacterized, genetic mutations that can cause the same pathological effect.

Here, we report a somatic missense mutation c.1061C >T (p.P354L) in the $N B N$ gene of a Chinese patient with CCS that did not harbor the typically expected EWSR1-ATF1 fusion. The pathogenesis of the $\sim 30 \%$ of CCS cases lacking an EWSR1 rearrangement has always been a mystery (19). The EWSR1-ATF1 fusion is one of the characteristics used to distinguish CCS from cutaneous melanoma (4). In contrast to most melanomas, CCS cells lack BRAF mutations (9) and show immunohistochemical positive staining for HMB45 and negative staining for $\mathrm{CK}(12,15)$. In our case study, the immunohistochemical results were consistent with the diagnostic criteria of CCS. Moreover, we observed a combination of cords and nests of clear and eosinophilic cells in a hyalinized background, which is a typical feature of CCS in histological diagnoses. Therefore, the finding of this novel $N B N$ mutation provides a novel genotypic feature for the clinical genetic diagnosis of CCS.

This mutation in $N B N$ was located within a phylogenetically conserved region, suggesting that it has a role of structural and/or functional importance. Previous studies have indicated that mutations in $N B N$ are associated with Nijmegen breakage syndrome, an autosomal recessive chromosomal instability syndrome characterized by microcephaly, growth retardation, immunodeficiency and cancer predisposition. The NBN protein is a member of the MRE11/ RAD50 double-strand break (DSB) repair complex, which consists of 5 proteins including NBN, MDC1, FANCD2, RRM2B and MRE11A (Fig. 6). This NBN protein is thought to be involved in DNA DSB repair and DNA damage-induced checkpoint activation (38). Cancers associated with NBN include gastrointestinal lymphoma (28), childhood acute leukemia (29), glioblastomas (30), breast cancer (25), prostate cancer (39) and ovarian cancer (40). Given these known associations, the mechanism of carcinogenesis for CCS may be related to or share similar pathway(s) associated with other NBN-related cancers. This intriguing supposition will require further investigation. In conclusion, our findings broaden the genotypic spectrum of CCS and provide new molecular insight that should prove valuable for the future clinical genetic diagnosis for CCS.

\section{Acknowledgements}

We thank all subjects for their participation. We also thank the scientists responsible for various web sources, including the 1000 Genomes Project (http://www.1000genomes.org), dbSNP (http://www.ncbi.nlm.nih.gov/SNP), ESP-6500 (http://evs.gs.washington.edu/EVS/), HapMap (http://hapmap. ncbi.nlm.nih.gov/) and the various DNA damage prediction tools including SIFT (http://sift.jcvi.org) and PolyPhen-2 (http://genetics.bwh.harvard.edu/pph2), among others.

\section{References}

1. Manoel EM, Reiser R, Brodskyn F, Franco M, Abrahão M and Cervantes O: Clear cell sarcoma of the parotid region. Rev Braz Otorrinolaringol 78: 135, 2012.

2. Ipach I, Mittag F, Kopp HG, Kunze B, Wolf P and Kluba T: Clear-cell sarcoma of the soft tissue - a rare diagnosis with a fatal outcome. Eur J Cancer Care (Engl) 21: 412-420, 2012.

3. Nwanyanwu KH, Comer G and Demirci H: Presumed choroidal metastasis secondary to clear cell sarcoma of the right knee. Int Ophthalmol 33: 163-165, 2013.

4. Meis-Kindblom JM: Clear cell sarcoma of tendons and aponeuroses: a historical perspective and tribute to the man behind the entity. Adv Anat Pathol 13: 286-292, 2006.

5. Wang WL, Mayordomo E, Zhang W, Hernandez VS, Tuvin D, Garcia L, Lev DC, Lazar AJ and López-Terrada D: Detection and characterization of EWSR1/ATF1 and EWSR1/CREB1 chimeric transcripts in clear cell sarcoma (melanoma of soft parts). Mod Pathol 22: 1201-1209, 2009. 
6. Malchau SS, Hayden J, Hornicek F and Mankin HJ: Clear cell sarcoma of soft tissues. J Surg Oncol 95: 519-522, 2007.

7. Mavrogenis A, Bianchi G, Stavropoulos N, Papagelopoulos P and Ruggieri P: Clinicopathological features, diagnosis and treatment of clear cell sarcoma/melanoma of soft parts. Hippokratia 17: 298-302, 2013.

8. Singh M, Ieremia E, Debiec-Rychter M, Connolly G and Calonje JE: Clear cell sarcoma of the tongue. Histopathology 64 : $750-751,2014$

9. Kraft S, Antonescu CR, Rosenberg AE, Deschler DG and Nielsen GP: Primary clear cell sarcoma of the tongue. Arch Pathol Lab Med 137: 1680-1683, 2013.

10. Lasithiotakis K, Protonotarios A, Lazarou V, Tzardi M and Chalkiadakis G: Clear cell sarcoma of the jejunum: a case report. World J Surg Oncol 11: 17, 2013.

11. Zhang W, Shen Y, Wan R and Zhu Y: Primary clear cell sarcoma of the sacrum: a case report. Skeletal Radiol 40: 633-639, 2011.

12. Sidiropoulos M, Busam K, Guitart J, Laskin WB, Wagner AM and Gerami P: Superficial paramucosal clear cell sarcoma of the soft parts resembling melanoma in a 13 -year-old boy. J Cutan Pathol 40: 265-268, 2013

13. Curry CV, Dishop MK, Hicks MJ, Naeem R, Reed JA and López-Terrada DH: Clear cell sarcoma of soft tissue: diagnostic utility of fluorescence in situ hybridization and reverse transcriptase polymerase chain reaction. J Cutan Pathol 35: 411-417, 2008.

14. Hisaoka M, Ishida T, Kuo TT, Matsuyama A, Imamura T, Nishida K, Kuroda H, Inayama Y, Oshiro H, Kobayashi H, et al: Clear cell sarcoma of soft tissue: a clinicopathologic, immunohistochemical, and molecular analysis of 33 cases. Am J Surg Pathol 32: 452-460, 2008

15. Davis IJ, Kim JJ, Ozsolak F, Widlund HR, Rozenblatt-Rosen O, Granter SR, Du J, Fletcher JA, Denny CT, Lessnick SL, et al Oncogenic MITF dysregulation in clear cell sarcoma: defining the MiT family of human cancers. Cancer Cell 9: 473-484, 2006

16. Yang L, Chen Y, Cui T, Knösel T, Zhang Q, Geier C, Katenkamp D and Petersen I: Identification of biomarkers to distinguish clear cell sarcoma from malignant melanoma. Hum Pathol 43: $1463-1470,2012$

17. Antonescu CR, Tschernyavsky SJ, Woodruff JM, Jungbluth AA Brennan MF and Ladanyi M: Molecular diagnosis of clear cell sarcoma: detection of $E W S-A T F 1$ and $M I T F-M$ transcripts an histopathological and ultrastructural analysis of 12 cases. J Mol Diagn 4: 44-52, 2002

18. Antonescu CR, Dal Cin P, Nafa K, Teot LA, Surti U, Fletcher CD and Ladanyi M: EWSR1-CREB1 is the predominant gene fusion in angiomatoid fibrous histiocytoma. Genes Chromosomes Cancer 46: 1051-1060, 2007.

19. Patel RM, Downs-Kelly E, Weiss SW, Folpe AL, Tubbs RR Tuthill RJ, Goldblum JR and Skacel M: Dual-color, break-apart fluorescence in situ hybridization for $E W S$ gene rearrangement distinguishes clear cell sarcoma of soft tissue from malignant melanoma. Mod Pathol 18: 1585-1590, 2005.

20. Antonescu CR, Katabi N, Zhang L, Sung YS, Seethala RR, Jordan RC, Perez-Ordoñez B, Have C, Asa SL, Leong IT, et al: EWSR1-ATF1 fusion is a novel and consistent finding in hyalinizing clear-cell carcinoma of salivary gland. Genes Chromosomes Cancer 50: 559-570, 2011.

21. Wang WL, Mayordomo E, Zhang W, Hernandez VS, Tuvin D, Garcia L, Lev DC, Lazar AJ and López-Terrada D: Detection and characterization of EWSR 1/ATF1 and EWSR1/CREB1 chimeric transcripts in clear cell sarcoma (melanoma of soft parts). Mod Pathol 22: 1201-1209, 2009

22. Gerlinger M, Rowan AJ, Horswell S, Larkin J, Endesfelder D, Gronroos E, Martinez P, Matthews N, Stewart A, Tarpey P, et al: Intratumor heterogeneity and branched evolution revealed by multiregion sequencing. N Engl J Med 366: 883-892, 2012.

23. Debniak T, Górski B, Cybulski C, Jakubowska A, Kurzawski G, Lener M, Mierzejewski M, Masojć B, Medrek K, Kładny J, et al: Germline 657del5 mutation in the NBS1 gene in patients with malignant melanoma of the skin. Melanoma Res 13: 365-370, 2003 .
24. Meyer P, Stapelmann H, Frank B, Varon R, Burwinkel B, Schmitt C, Boettger MB, Klaes R, Sperling K, Hemminki K, et al: Molecular genetic analysis of NBS1 in German melanoma patients. Melanoma Res 17: 109-116, 2007.

25. Desjardins S, Beauparlant JC, Labrie Y, Ouellette G, Durocher F; INHERIT BRCAs: Variations in the NBN/NBS1 gene and the risk of breast cancer in non-BRCA1/2 French Canadian families with high risk of breast cancer. BMC Cancer 9: 181, 2009.

26. Jazayeri A, Balestrini A, Garner E, Haber JE and Costanzo V: Mre11-Rad50-Nbs1-dependent processing of DNA breaks generates oligonucleotides that stimulate ATM activity. EMBO J 27: 1953-1962, 2008.

27. Varon R, Vissinga C, Platzer M, Cerosaletti KM, Chrzanowska KH, Saar K, Beckmann G, Seemanová E, Cooper PR, Nowak NJ, et al: Nibrin, a novel DNA double-strand break repair protein, is mutated in Nijmegen breakage syndrome. Cell 93: 467-476, 1998.

28. Steffen J, Maneva G, Popławska L, Varon R, Mioduszewska O and Sperling K: Increased risk of gastrointestinal lymphoma in carriers of the 657del5 NBS1 gene mutation. Int J Cancer 119: 2970-2973, 2006.

29. Mosor M, Ziółkowska I, Januszkiewicz-Lewandowska D and Nowak J: Polymorphisms and haplotypes of the NBSI gene in childhood acute leukaemia. Eur J Cancer 44: 2226-2232, 2008.

30. Watanabe T, Nobusawa S, Lu S, Huang J, Mittelbronn M and Ohgaki $\mathrm{H}$ : Mutational inactivation of the nijmegen breakage syndrome gene $(N B S 1)$ in glioblastomas is associated with multiple TP53 mutations. J Neuropathol Exp Neurol 68: 210-215, 2009.

31. Xiu X, Yuan J, Deng X, Xiao J, Xu H, Zeng Z, Guan L, Xu F and Deng S: A novel COL4A5 mutation identified in a Chinese Han family using exome sequencing. BioMed Res Int 2014: 186048, 2014.

32. Gilissen C, Arts HH, Hoischen A, Spruijt L, Mans DA, Arts P, van Lier B, Steehouwer M, van Reeuwijk J, Kant SG, et al: Exome sequencing identifies WDR35 variants involved in Sensenbrenner syndrome. Am J Hum Genet 87: 418-423, 2010.

33. Guo Y, Yuan J, Liang H, Xiao J, Xu H, Yuan L, Gao K, Wu B, Tang Y, Li X, et al: Identification of a novel COL4A5 mutation in a Chinese family with X-linked Alport syndrome using exome sequencing. Mol Biol Rep 41: 3631-3635, 2014.

34. ENCODE Project Consortium: The ENCODE (ENCyclopedia Of DNA Elements) Project. Science 306: 636-640, 2004.

35. Zhou VW, Goren A and Bernstein BE: Charting histone modifications and the functional organization of mammalian genomes. Nat Rev Genet 12: 7-18, 2011.

36. Bianchi G, Charoenlap C, Cocchi S, Rani N, Campagnoni S, Righi A, Frisoni T and Donati DM: Clear cell sarcoma of soft tissue: a retrospective review and analysis of 31 cases treated at Istituto Ortopedico Rizzoli. Eur J Surg Oncol 40: 505-510, 2014.

37. Nakayama S, Yokote T, Iwaki K, Akioka T, Miyoshi T, Hirata Y, Takayama A, Nishiwaki U, Masuda Y, Tsuji M, et al: A rare case of primary clear cell sarcoma of the pubic bone resembling small round cell tumor: an unusual morphological variant. BMC Cancer 12: 538, 2012

38. Huang J, Grotzer MA, Watanabe T, Hewer E, Pietsch T, Rutkowski S and Ohgaki H: Mutations in the Nijmegen breakage syndrome gene in medulloblastomas. Clin Cancer Res 14: 4053-4058, 2008.

39. Zuhlke KA, Johnson AM, Okoth LA, Stoffel EM, Robbins CM, Tembe WA, Salinas CA, Zheng SL, Xu J, Carpten JD, et al: Identification of a novel NBN truncating mutation in a family with hereditary prostate cancer. Fam Cancer 11: 595-600, 2012.

40. Porhanova NV, Sokolenko AP, Sherina NY, Ponomariova DN, Tkachenko NN, Matsko DE and Imyanitov EN: Ovarian cancer patient with germline mutations in both $B R C A 1$ and $N B N$ genes. Cancer Genet Cytogenet 186: 122-124, 2008. 